\title{
Index of Subjects for Volume 11
}

\author{
Aborigines, Australjan, diet 5-23 \\ absorption from small intestine in dog 61 \\ acidosis in rumen 186 \\ acids, digestive, in dog 49-50, 58-60 \\ activity, physical see exercise \\ ammonia see nitrogen \\ amylase in digestion, dog $48,55-56$ \\ anorexia nervosa see eating disorders \\ antinutrients eaten by Aborigines 18-19 \\ antinutritional factors, effects in feeds for pigs and \\ poultry 91-114 \\ Australia see Aborigines, Australian
}

bacteria, ruminal $175-176$

bacteriophages, ruminal 177

behaviour, foraging $25-43,199-222,225-231$

beverages drunk by Aborigines 18

bile acids in irritable bowel syndrome 299

bile in dog 55-58

blood pressure, effects of activity, rat 74

bulimia see eating disorders

carbohydrates, ruminal digestion 178-180

cardiovascular disease see vascular diseases

cellulose see carbohydrates

cereals, sources of zinc 123

children, physical activity and nutrition, effects $71-90$

chalesterol in human milk 258

in infant formulas 269-271

chymotrypsin in digestion, dog 55,57

chytridiomycetes in ruminants 133-168

connective tissue disease, effects of homocysteine 330-331

cystathione $\beta$-synthase 316,327

defaunation of ruminal protozoa 176

effects on numinal fungi $149-150$

diabetes, effects of homocysteine 331

diabetes mellitus, non-insulin dependent, effect of Aborigine diet 19-20

diet, surveys, under-reporting 231-253

dog, gut, modelling of physiology $\quad 45-69$

eating disorders in irritable bowel syndrome 294-295

electrolytes in physiology of dog $48-49,56,58-60$

energy, output in children $80-84$

enzymes, digestive, in dog $47-49,53-56$

exercise during pregnancy, rat $76-77$

exercise in young, effects $71-90$

fatty acids see also lipids

in infant formulas 262-269 fermentation, ruminal, manipulation 189-190

fibre, dietary, in irritable bowel syndrome 291-292

fluoroacetate, poison in ruminants 188

folate, effects of nutritional deficiency $318-320$

foods, animal learning and motivation $25-43$

Australian Aboriginal 5-23

imbalance in irritable bowel syndrome 292-294

sensitivities in irritable bowel syndrome 281-300

foraging behaviour $25-43,199-222,225-231$

fruits eaten by Aborigines 8-9

fungi see also chytridiomycetes

manipulation of populations in numen $156-159$

ruminal 177

gall bladder emptying in dog 55-59

gastric emptying in dog $50-56$

gastrointestinal tract see gut

gene transfer into ruminal bacteria $179-180$

$\beta$-glucanases, additives in feeds for pigs and poultry 94-101

$\beta$-glucans, structure 93

growth and development, effects of activity and nutrition, rat 74-75

effects of early nutrition, children 79

gut see also irritable bowel syndrome, inflammatory bowel disease

flora, effects on added feed enzymes, pigs and poultry 101

differences between pigs and poultry $\quad 92-93$

hare, pregnant, effects of exercise 77

heart, disease, effect of Aborigine diet 19-20

effects of exercise and nutrition in children $80-81$ young rats $73-76$

homocysteine, biochemistry $312-320$

risk factor for vascular diseases 311-338

structure and properties 312--315

infection, microbial, in irritable bowel syndrome $299-300$

inflammatory bowel disease, effects of homocysteine 331

intestinal transit in dog 62-65

intestine, small, physiology in dog 58-65

irritable bowel syndrome $279-310$

kidney see renal failure

lactic acid, ruminal production 180

lactose intolerance in irritable bowel syndrome 290-291

leaves eaten by Aborigines 17-18

linoleic acid in infant formulas 263-264, 271 
$\alpha$-linolenic acid in infant formulas

lipase in digestion, dog 48, 53-57

lipases, hurnan 259

lipids, digestion in infants 259-261

human milk, composition 256-258

infant formulas $255-278$

metabolism, young rat 75,77

ruminal metabolism $180-181,184-186$

litter size, effects on development in rats 73

markers, ruminal digestion 183

methane, numinal production 186

methionine synthase $\quad 316-317$

5,10-methylenetetrahydrofolate reductase $316,327-330$

mimosin, toxic in rumen 187

minerals eaten by Aborigines 18

monensin, effect on ruminal fungi 150

ruminal metabolism 189

mothers, undemourished in pregnancy, effects on children 77-79

effects on young rats 74

mycotoxins, ruminal degradation 187

myo-inositol hexaphosphate see phytic acid

neural tube defect see vascular diseases

nitrates, ruminant poisons 187

nitrogen, ruminal metabolism 181-182

nutrition in young, effects 71-90

nuts eaten by Aborigines 9-15

obesity, dietary under-reporting 238, 240-242

effect of Aborigine diet 19-20

oxalate, ruminal degradation 187

palaeolithic nutrition $21-22$

palatability in foraging behaviour $209-211$

pancreas, physiology in dog 53-55

pepsin in digestion, dog 48

phenolics, effects on numinal fungi 152-153

phospholipids in human milk 258

phosphorus, inorganic, effects of phytase in feeds for pigs and poultry 104

phytase, additive in feeds for pigs and poultry 101-105

phytic acid, effect on zinc absorption 123-127

pigs, enzyme additives in feedstuffs 91-114

polyoxins, effects on ruminal fungi 151

polyphenols, toxic in rumen 188

polysaccharides, ruminal digestion $\quad 178-181$ poultry, enzyme additives in feedstuffs 91-114 propionate, effect on ruminal fungi 151 protein see also nitrogen

effects of ruminal fungi 155-156

microbial synthesis in rumen 183

nutrition, effects in children $79-80$

protozoa, numinal 176-177

psychosomatics in food sensitivities 289-290

purpose in foraging behaviour 25-43, 223-229

rat, young, physical activity and nutrition, effects $71-90$

renal failure, chronic, effects of homocysteine 330

roots and tubers eaten by Aborigines 9-15

numen, metabolism 173-198

ruminants, chytridiomycetes 133-168

salinomycin, effect on ruminal fungi 151

saliva, dog $47-48$

sapogenins, toxic in rumen 187

seeds eaten by Aborigines 15-17

self-organization in living organisms 199-222

starch see carbohydrates

stomach, physiology, dog 48-53

stress in intitable bowel syndrome 295-298

sulphur, dietary, effect on ruminal fungi 147

tetronasin, effect on ruminal fungi 150

toxins, ruminal 187-189

triacylglycerols in human milk 256, 259-260

in infant formulas 261-262

trypsin in digestion, dog 56

urea see also nitrogen

vascular diseases, homocysteine as risk factor 311-338

viscosity of digesta in pigs and poultry $97-100$

xylanases, additives in feeds for pigs and poultry 93-101

xylans, structure 93

yeasts, ruminal metabolism 189

zinc, absorption enhancers 123

deficiency in developing countries 115-131 